\title{
Serum Levels of IGF-1 and IGFBP-3 in Relation to Clinical and Pathobiological Aspects of Head and Neck Squamous Cell Carcinomas
}

\author{
DAVID KALFERT ${ }^{1,2}$, MARIE LUDVIKOVA $^{3}$, ONDREJ TOPOLCAN ${ }^{4}$, PETR CELAKOVSKY $^{2}$, \\ RADEK KUCERA ${ }^{4}$, JINDRA WINDRICHOVA ${ }^{4}$, JAROSLAV LUDVIK ${ }^{5}$, \\ KATERINA SKALOVA ${ }^{2}$, VLASTIMIL KULDA ${ }^{6}$, MARTIN PESTA $^{3}$ and JAN PLZAK ${ }^{1}$ \\ ${ }^{1}$ Department of Otorhinolaryngology and Head and Neck Surgery, University Hospital Motol, \\ First Faculty of Medicine, Charles University, Prague, Czech Republic; \\ ${ }^{2}$ Department of Otorhinolaryngology and Head and Neck Surgery, University Hospital Hradec Kralove, \\ Faculty of Medicine in Hradec Kralove, Charles University, Prague, Czech Republic; \\ Departments of ${ }^{3}$ Biology, ${ }^{6}$ Medical Chemistry and Biochemistry, \\ Faculty of Medicine in Pilsen, Charles University, Prague, Czech Republic; \\ Departments of ${ }^{4}$ Immunochemistry, ${ }^{5}$ Imaging Methods, University Hospital Pilsen, \\ Faculty of Medicine in Pilsen, Charles University, Prague, Czech Republic
}

\begin{abstract}
Background/Aim: Head and neck squamous cell carcinoma (HNSCC) includes tumors of various anatomical sites sharing multifactorial etiopathogenesis and generally dismal response to conventional treatment. The objective of this study was to determine the clinical significance of serum levels of insulin-like growth factor-1 (IGF-1) and insulin-like growth factor-binding protein-3 (IGFBP-3) in HNSCC. Patients and Methods: A total of 46 patients, with histologically-confirmed diagnosis of HNSCC (21 oropharyngeal, 21 laryngeal, and 4 hypopharyngeal cancers) were enrolled in this study. IGF-1 and IGFBP-3 serum levels were measured by an immunoradiometric assay using commercial kits. The adjustment of serum levels at 60 years of age was performed. Results: Significant differences were found in IGF-1 serum concentrations between patients with 16 positive and p16 negative HNSCC $(p=0.0062)$, with higher IGF-1 levels in p16 positive tumors, between lowgrade and high-grade cancers $(p=0.0323)$ only in larynx, with elevated IGF-1 concentrations associated with highgrade and between recurrent and non-recurrent HNSCC $(p=0.0354)$, with lower IGF-1 levels in recurrent tumors.
\end{abstract}

Correspondence to: Marie Ludvikova, Department of Biology, Faculty of Medicine in Pilsen, Charles University, Alej Svobody 1655/76, 30460 Pilsen, Czech Republic. Tel: +420 378024270, email:ludvikova.m@mail.cz and marie.ludvikova@lfp.cuni.cz

Key Words: Head and neck squamous cell carcinoma, IGF-1, IGFBP-3, serum tumor marker, p16.
Conclusion: The conflicting results of this study may reflect some abnormality of IGF axis regulation in HNSCC, as well as the influence of other etiological factors (e.g. smoking, HPV infection).

Head and neck squamous cell carcinomas (HNSCC) represent, in spite of the same histopathological type, a heterogeneous group of tumors in respect to their different site of origin, etiology and pathobiology, clinical behavior and prognosis (1). Smoking habit, alcohol abuse and human papillomavirus (HPV) infection are generally acknowledged to be etiological factors of HNSCC (2). p16 Protein is supposed to be a robust marker for HPV-induced oncogenesis, as it is overexpressed in HPV-positive tumors (3-5). Despite advancements in therapeutic strategies for the treatment of HNSCC, prognosis has not shown any improvement during the last decades, with many cases of recurrent disease. A better understanding of the pathobiology of distinct site-specific types of HNSCC and the identification of novel molecules playing the role of prognostic and predictive biomarkers might help to improve early detection, prognosis and therapeutic options for these tumor types.

Increased levels of insulin-like growth factor-1 (IGF-1) and decreased levels of insulin-like growth factor-binding protein-3 (IGFBP-3) or an increase in the ratio of IGF-1 to IGFBP-3 have been reported to be related to hyperproliferation, development and progression in several common cancers, including breast, prostate, lung, colon and esophagus etc. $(6,7)$. However, the role of both of these 
Table I. The clinicopathological profile of the study population.

\begin{tabular}{|c|c|c|c|c|c|}
\hline Clinical variables & $\mathrm{N}$ & $\%$ & Prognostic variables & $\mathrm{N}$ & $\%$ \\
\hline Gender & & & Histological grading & & \\
\hline Male & 38 & 82.6 & G1-2 & 32 & 69.6 \\
\hline Female & 8 & 17.4 & G3 & 14 & 30.4 \\
\hline Age (years) & & & TNM staging ${ }^{\mathrm{a}}$ & & \\
\hline$\leq 60$ & 28 & 60.9 & I-II & 13 & 28.3 \\
\hline$>60$ & 18 & 39.1 & III-IV & 33 & 71.7 \\
\hline Anatomic site & & & Local recurrenceb & & \\
\hline Oropharynx and hypopharynx & $25^{*}$ & $54.3 *$ & No & 33 & 71.7 \\
\hline Larynx & 21 & 45.7 & Yes & 13 & 28.3 \\
\hline Smoking habit & & & p16 status & & \\
\hline Non-smoker & 14 & 30.4 & Positive & 17 & 37.0 \\
\hline Smoker & 32 & 69.6 & Negative & 29 & 63.0 \\
\hline
\end{tabular}

aTNM Classification of Malignant Tumours, 7th edition (10); bfollow-up: 12-64 months; *inclusive hypopharyngeal cancers -4 cases (8.6\%).

markers in cancer is variable and not fully understood with conflicting evidences in the literature (8). The impact of IGF family in HNSCC and its potential for novel IGF therapies is unclear (9). Hence, we have been prompted to shed light on the IGF-1 and IGFBP-3 expression in HNSCC patients overall and with respect to the selected locations and the potential clinical consequences and interactions.

\section{Patients and Methods}

Patient population. A total of 46 patients with histologically proven diagnosis of HNSCC treated at the Department of Otorhinolaryngology and Head and Neck Surgery, University Hospital in Hradec Kralove were enrolled in the study that began in July 2011 and closed for new patients accrual in December 2012. Clinicopathological data and follow-up information for all the patients were available. Both smokers and non-smokers were included in the study. Patients with other malignancies, inflammatory and infectious diseases were excluded. The study group comprised of HNSCCs of different subsites: oropharyngeal (21 cases), hypopharyngeal (4 cases), and laryngeal (21 cases). Tumor grade was evaluated following World Health Organization (WHO) criteria and tumor staging was determined using the TNM system of the International Union Against Cancer (10). Patients were grouped into stage I-II and stage III-IV. Standard practice in our University Hospital is the determination of the protein p16 expression status in HNSCC as an integral part of the final histological diagnosis. Data relevant to grading, staging, p16 status and other characteristics of cancers were retrieved from the hospital's information system. All participants were informed about the research study and written informed consent was obtained from each patient. The study was approved by the Ethics Committee, University Hospital Hradec Kralove (number 201105 $\mathrm{S} 14 \mathrm{P})$. Treatment decision-making was based on the clinical status of patients and on the grading and staging of tumors. All the patients underwent surgery followed with radiotherapy or chemoradiotherapy. The clinicopathological characteristics of the study populations are shown in Table I.
Blood serum collection and procedures. Ten milliliters of peripheral blood were drawn from every patient before surgery and from 28 patients 3 months after completion of treatment from 7 to 9 a.m. using standardized phlebotomy procedures. Blood samples were collected without anticoagulant into red top vacutainers and allow to coagulate for 20 to $30 \mathrm{~min}$ at room temperature. Sera were separated by centrifugation of blood for $10 \mathrm{~min}$ at $1700 \mathrm{~g}$. All specimens were immediately aliquoted and stored at $-80^{\circ} \mathrm{C}$ until the time of analysis.

Sample analysis. IGF-1 serum levels were measured by an Immunoradiometric assay (IRMA) using the commercial kit IRMA IGF-1 (Immunotech Beckman Coulter Company, Prague, Czech Republic), with interassay Coefficient of variation (CV) of $6.8 \%$ at a concentration of $25.0 \mathrm{ng} / \mathrm{ml}$, and analytical sensitivity of $2.0 \mathrm{ng} / \mathrm{ml}$. The calibrators in the Immunotech IGF-1 assay were calibrated against the international reference standard, WHO 87/518.

IGFBP-3 serum levels were measured using the radioimmunoassay kit IRMA IGFBP-3 (DIAsource, Belgium), with interassay $\mathrm{CV}$ of $6.23 \%$ at a concentration of $61.5 \mathrm{ng} / \mathrm{ml}$, and analytical sensitivity of $0.27 \mathrm{ng} / \mathrm{ml}$. Both analyses were performed with the Stratec SR 300 instrument.

Adjustment for age. The adjustment of serum levels at 60 years of age was performed to avoid the misleading results of IGF-1 an IGFBP-3 analyses. The age-specific reference values for serum IGF1, IGFBP-3 and IGF-1/IGFBP-3 ratio was established in healthy Czech adults by using the linear regression model published by Kucera et al. served as a guideline of the adjustment (11).

Statistical analysis. The results obtained in our study were statistically analyzed. The results for the subjects were given as the mean \pm S.D. and median. Statistical significance between the different variables was determined with the Spearman's rank order. The Wilcoxon two-sample test and Kruskal-Wallis test were used for estimation of statistical significance for association between serum levels of IGF-1 and IGFBP-3 and clinicopathological parameters. $p$-Values less than 0.05 were considered as statistically significant. Statistical analysis was carried out using SAS 9.23 
Table II. Comparison of IGF-1 serum levels in relation to selected clinicopathological variables of HNSCC.

\begin{tabular}{lrrr}
\hline Parameter & N $\begin{array}{r}\text { IGF-1 serum levels } \\
\text { (adjusted for age) }\end{array}$ \\
\cline { 2 - 5 } & & $\begin{array}{r}\text { IGF-1 } \\
\text { level }\end{array}$ & $p$-Value \\
& & & \\
& & & \\
Grade (laryngeal cancer) & 6 & $\downarrow$ & 0.0323 \\
$\quad$ G1-2 & 15 & $\uparrow$ & \\
$\quad$ G3 & & & \\
p16 status & 17 & $\uparrow$ & 0.0062 \\
$\quad$ Positive & 29 & $\downarrow$ & \\
$\quad$ Negative & & & \\
Recurrence of HNSCC irrespective of subsites & 13 & $\downarrow$ & 0.0354 \\
$\quad$ Yes & 33 & $\uparrow$ & \\
$\quad$ No & & & \\
Recurrence of oropharyngeal cancer & 5 & $\downarrow$ & 0.0101 \\
$\quad$ Yes & 16 & $\uparrow$ & \\
$\quad$ No & & & \\
Therapy & 46 & $\downarrow$ & 0.0231 \\
$\quad$ Before* & & \\
After* & 28 & $\uparrow$ & \\
$\quad$
\end{tabular}

Abbreviation: $\uparrow$ increased; $\downarrow$ decreased. *Peripheral blood was drawn from every patient before surgery and from 28 patients 3 months after completion of treatment.

software (Statistical Analysis Software release 9.3. SAS Institute Inc., Cary, North Carolina, USA).

\section{Results}

The median age at the time of diagnosis was 60 years (men 59.5 , women 60) with range 46-85 years of age. IGF-1 and IGFBP-3 levels decreased continuously with age. Serum levels of studied markers before and after the adjustment for the age of 60 years were statistically evaluated. IGF-1 serum concentrations without adjustment were statistically significantly higher in patients of $\leq 60$ years of age $(p=0.0363)$, while no differences in IGF-1 levels adjusted on age were revealed between age categories $\leq 60$ and $>60$ years $(p=0.9481)$. All other statistical analyses, comparisons and correlations, were performed with the adjusted data (Table II).

Comparison of studied marker rates in relation to clinical variables of HNSCC patients. No significant differences in the serum levels of IGF-1 and IGFBP-3, respectively, were found in respect to selected clinicopathological variables, e.g. sex (male vs. female), anatomic site (oropharyngeal versus hypopharyngeal versus laryngeal), abuses (smokers versus non-smokers), and TNM staging (stage I-II versus stage III-IV). Only in laryngeal tumors significant differences were found in adjusted IGF-1 levels between men and women, the latter had elevated IGF-1

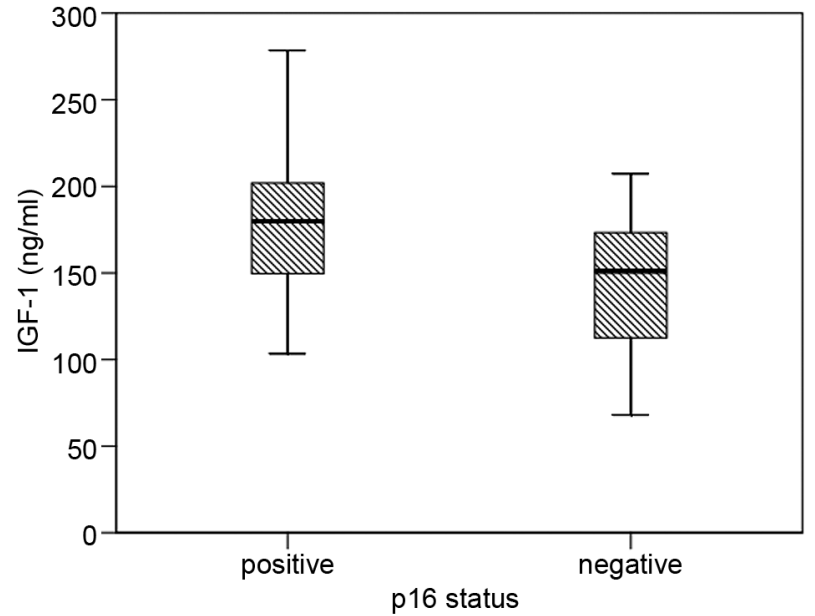

Figure 1. Relationship of IGF-1 serum levels and p16 status (p16 positive versus p16 negative).

concentrations $(p=0.0159)$. No statistically significant differences between serum IGF-1 and IGFBP-3 levels were found in HNSCC patients.

Comparison of studied markers in relation to p16 status of $H N S C C$. Statistically significant differences were found in IGF-1 serum concentrations between patients with p16 positive and p16 negative HNSCC before and after IGF-1 levels adjustment ( $p=0.0197 ; p=0.0062$, respectively), with higher IGF-1 levels in p16 positive tumors (Figure 1; Table II). No differences were found in IGFBP-3 serum levels and IGF-1/IGFBP-3 ratio, respectively, between p16 positive and p16 negative HNSCC.

Comparison of studied marker in relation to prognostic parameters of HNSCC. Studied markers were analyzed in relation to the prognostic parameters of HNSCC, notably grading, recurrence, p16 status as well as to timing (beginning and completion) of therapy (or treatment) (Table II). In respect to the grade of tumors, IGF-1 levels adjusted on age showed significant differences between low-grade (G1, G2) and high-grade (G3) cancers only in larynx, with elevated IGF-1 concentrations associated with grade G3 $(p=0.0323)$. Oropharyngeal low-grade and high-grade cancers did not show any differences in IGF-1 expression.

Taking all cancers together, recurrent HNSCC showed significantly lower age-adjusted IGF-1 levels as well as IGF$1 /$ IGFBP-3 ratio compared with those without recurrence ( $p=0.0364 ; p=0.0495$, respectively). Focusing on sitespecific HNSCC, only oropharyngeal cancers (not laryngeal) were associated with significant differences in IGF-1 levels between recurrent and non-recurrent tumors $(p=0.0101)$. 
Statistically significant correlation was confirmed between IGF-1 levels before and after treatment of HNSCC ( $\mathrm{r}=0.51$, $p=0.0072)$ with significantly elevated IGF-1 levels after HNSCC treatment ( $p=0.0075 ; p=0.0231$, respectively).

The significant relation of p16 expression to behavior of HNSCC (recurrent versus non-recurrent tumors) was confirmed in oropharyngeal cancers with better behavior without recurrence in 16 positive tumors $(p=0.0075)$.

\section{Discussion}

Levels of IGF-1 and IGFBP-3 are quite stable in circulation with a gradual fall with age after puberty and with different dynamics of the decline between females and males (11-13). Adjustment of IGF-1 and IGFBP-3 for age, as mentioned above, made it possible to perform more reliable interpretation of results.

The IGF-1 signaling pathway regulates critical cellular processes including differentiation, homeostasis and aging. It involves IGF-1 ligand, transmembrane IGF-1R receptor and downstream signaling molecules of two signaling branches: PI3K-PDK1-AKT and RAS-RAF-MAPK pathways. The dysregulation of IGF axis and its role in promoting cancer has been investigated for many years (14). The higher serum levels of IGF-1 and lower of IGFBP-3 are generally considered to be linked to an increased risk, progression and mortality of many malignant tumors, namely breast and ovarian cancers $(8,15$, 16), prostate (17) and nasopharyngeal carcinomas (18). Moreover, IGF axis can induce tumor-associated lymphangiogenesis and promote lymphatic metastasis (19). The monitoring of serum IGF-1 and IGFBP-3 levels was supposed to be an important clinical tool and an emerging diagnostic and prognostic marker (20). Regarding HNSCC, a limited number of studies have focused on the serum and tumor tissue IGF-1 and IGFBP-3 levels (21). Although site-specific HNSCC may have different pathobiologies (notably oropharyngeal and laryngeal cancers), the majority of existing studies do not take into account their different subsites of origin or alternatively squamous cell carcinomas of the oral cavity and tongue usually distinctly prevail in published papers $(9,22)$. In our cohort, we aimed to make provision for tumor location, but no significant differences in the serum levels of IGF-1 and IGFBP-3 between separate anatomic sites were revealed. In contrast to the logically increased IGF-1 and decreased IGFBP-3 levels in many cancers, lots of conflicting results are known in HNSCC (8, 15-18). Zhi et al. have described the trend for downregulation IGF-1, 2 and up-regulation of IGFPB-3 mRNA in laryngeal cancer and Brady et al. reported lower circulating levels of both IGF-1 and IGFBP-3 in patients with oral cancers suggesting different pathobiology of carcinoma in these particular locations $(21,22)$. Additionally, Limesand et al. published the controversial correlations (both positive and negative) between IGF-1/IGF-1R levels and clinical outcomes of HNSCC (9). The above mentioned conflicting evidence on the pattern of IGF-1/IGFBP-3 expression are in concordance with some of our findings of significantly higher IGF-1 serum levels in tumors after therapy, in p16 positive cancers and with significantly lower IGF-1 expression in recurrent HNSCC (9, 21, 22). However, Wu et al. in 2004 have found in their study the potential role of the elevated serum levels of IGF-1 and the deregulated (high or low) IGFBP-3 levels as predictors of the risk to develop second primary tumors in the patients with HNSCC (23). Although the majority of published studies are focused on IGFBP-3, recently there is a growing interest in IGFBP-5 levels and its suppressive effect on the carcinogenesis in HNSCC (24).

p16 positivity in HNSCC is consider to be a surrogate marker of the high-risk HPV (HR-HPV) infection and p16 positive oropharyngeal HNSCCs are known to have a better prognosis $(3,5,25,26)$. Therefore in our cohort of HNSCC, p16 status was established instead of HPV positivity/ negativity. We found significant correlation between $\mathrm{p} 16$ positivity and oropharyngeal carcinomas without recurrence $(p=0.0075)$. However, the significant correlation between elevated IGF-1 serum levels and p16 positive tumors in our study is contradictory to expected better prognosis of these tumors (27). Therefore the interactions between HPV/p16 and members of IGF family and their specific role in pathogenesis and progression of HNSCC require the further examination. In the most common HR-HPV related cancers, cervical and HNSCC, differential expressions within IGF pathway are evident (28). Taking together, it is our hypothesis that the interactions between IGF family and viral infections exist and seem to be tumor and virus specific.

In respect to the IGF axis and cigarette smoking habit, Kaklamani et al. have shown a significant reduction in the mean IGFBP-3 concentrations in cigarette smokers (29). No similar study of the relationship between the expression of IGF family members and smoking in patients with HNSCC was found in the literature. IGF-1 and IGFBP-3 levels did not show any significant differences between HNSCC patients - smokers and non-smokers in our study.

Notwithstanding that the IGF system was confirmed to play a key signaling role in the development and progression of many types of cancer (14), the significance of IGF machinery in HNSCC and other malignancies is controversial (20). Moreover, the assessment and comparison of some results are difficult since they are influenced by the size of the study populations, the study design as well as cancer sites (20). In addition, some of the tumors have shown missing or inverse correlation between IGF-1 levels and risk of cancer development and progression $(30,31)$. This fact might be explained by numerous external and internal influences modifying the IGF axis efficiency, notably microRNAs, genetic and epigenetic events and crosstalk between IGF-1 system and estrogens etc. $(32,33)$. Further impacts, namely viral infection, alcohol consumption and cigarette 
smoking are supposed to influence IGF-1 serum levels with ambiguous results $(17,34)$. In respect to viral infection, the interference between viruses, namely hepatitis $\mathrm{C}$ virus (HCV), and human papillomaviruses (HPVs), and IGF axis have been documented $(28,35,36)$. Mechanisms of the IGF deregulation in HCV/HPV associated cancer have not been fully understood $(28,35,36)$. Briefly, the relationship between IGF-1 family members and different stages of chronic hepatitis $\mathrm{C}$, liver cirrhosis and $\mathrm{HCV}$-associated hepatocellular carcinogenesis (HCC) has been studied and decreased tissue IGF-1 expression in all pathologic processes has been revealed $(37,38)$. In HCC, the increased cell proliferation was associated with increased synthesis of IGF-2 and IGF-1R activation probably mediated by $\mathrm{HCV}$ protein that could have a role in different splicing profiles of IGF-1 genes (36). Similarly, an interplay between IGF members family and oncogenic HR-HPV infection has been described. In the uterine cervix surprisingly the increased IGF-1 serum levels were significantly associated with reduced risk of HR-HPV cervical cancer transformation. This effect is hard to explain $(17,39)$. Mannhardt et al. confirmed that the E7 oncoprotein produced by the HR-HPV inactivates the IGFBP-3 in circulation and consequently supports the up-regulation of IGF-1 levels (40). Polanska et al. recently presented the results of their study focused on the effect of HPV on expression levels of selected tumor markers in HNSCC. Significantly higher expression of MMP9 $(p=0.02)$, VEGFA $(p=0.02)$, and other markers was found in HPV-negative tumor tissues (41). Although IGF-1 analysis was not included in their study, the deregulations of IGF family expression seem to be also very probable in HPV infected tumors. The detailed investigation of the IGF axis could provide new insight into the pathobiology of HNSCC with respect to the patient/tumor characteristics and the potential future perspectives for novel IGF therapies to extend current molecular - targeted treatment options $(42,43)$.

\section{Conclusion}

In conclusion, many controversial or non-significant results described in our study as well as those presented by other authors indicated a less clear role of IGF axis in the pathogenesis and progression of HNSCC. We are aware of some limitations of our study (e.g. small number of samples, the short follow-up time) that may have influenced results. We made an attempt to eliminate some drawbacks and to standardize the study by adjustment and detailed clinicopathological analysis of the HNSCC cohort. However, it is our hypothesis that the contradictory and ambiguous results of the present study may also reflect some abnormality of IGF axis regulation in HNSCC and the influence of other etiopathological factors (e.g. smoking, HPV infection). Therefore larger scale are required to determine the exact role of the IGF axis and serum IGF-1 and IGFBP-3 levels and to evaluate their promising emerging prognostic and predictive potential in HNSCC.

\section{Conflicts of Interest}

The Authors declared no potential conflicts of interest.

\section{Acknowledgements}

This study was supported by research projects of Charles University, Czech Republic GAUK No. 444311, by the Charles University Research Fund (project number P36), by research project of the Ministry of Health (Czech Republic) No. 00669806 (Faculty Hospital Plzen), and the Charles University research program PROGRES Q28/LF1.

\section{References}

1 Barnes L, Eveson JW, Reichart P and Sidransky D: WHO Classification Head and Neck Tumours Pathology \& Genetics Head and Neck Tumours. Lyon: IARC Press, 2005.

2 Klozar J, Tachezy R, Rotnaglova E, Koslabova E, Salakova M and Hamsikova E: Human papillomavirus in head and neck tumors: epidemiological, molecular and clinical aspects. Wien Med Wochenschr 160: 305-309, 2010.

3 Klozar J and Tachezy R: What are the implications of human papillomavirus status in oropharyngeal tumors for clinical practice? Curr Opin Otolaryngol Head Neck Surg 22: 90-94, 2014.

4 Thomas J and Primeaux T: Is p16 immunohistochemistry a more cost-effective method for identification of human papilloma virus-associated head and neck squamous cell carcinoma? Ann Diagn Pathol 16: 91-99, 2012.

5 Kalfert D, Celakovsky P, Laco J and Ludvikova M: The role of protein p16(INK4a) in glottic laryngeal squamous cell carcinoma. Pathol Oncol Res 20: 909-915, 2014.

6 Macaulay VM: Insulin-like growth factors and cancer. Br J Cancer 65: 311-320, 1992.

7 Yilmaz O, Eroglu A, Dag E, Karaoglanoglu N and Yilmaz A: Serum levels of IGF-I and IGFBP-III and their relation with carcinoembryonic antigen and carbohydrate antigen 19-9 in cases of esophageal cancer. Int J Clin Pract 60: 1604-1608, 2006.

8 Tas F, Karabulut S, Bilgin E, Tastekin D and Duranyildiz D: Clinical significance of serum insulin-like growth factor-1 (IGF1) and insulin-like growth factor binding protein-3 (IGFBP-3) in patients with breast cancer. Tumour Biol 35: 9303-9309, 2014.

9 Limesand $\mathrm{KH}$, Chibly AM and Fribley A: Impact of targeting insulin-like growth factor signaling in head and neck cancers. Growth Horm IGF Res 23: 135-140, 2013.

10 Sobin LH, Gospodarowicz MK and Wittekind C: TNM Classification of Malignant Tumours, Seventh Edition. WileyBlackwell, 2009.

11 Kucera R, Topolcan O, Pecen L, Kinkorova J, Svobodova S, Windrichova J and Fuchsova R: Reference values of IGF1, IGFBP3 and IGF1/IGFBP3 ratio in adult population in the Czech Republic. Clin Chim Acta 444: 271-277, 2015.

12 Lin CM, Huang YL and Lin ZY: Influence of gender on serum growth hormone, insulin-like growth factor-I and its binding protein-3 during aging. Yonsei Med J 50: 407-413, 2009.

13 Aimaretti G, Boschetti M, Corneli G, Gasco V, Valle D, Borsotti M, Rossi A, Barreca A, Fazzuoli L, Ferone D, Ghigo E and Minuto F: Normal age-dependent values of serum insulin growth factor-I: results from a healthy Italian population. J Endocrinol Invest 31: 445-449, 2008. 
14 Bowers LW, Rossi EL, O'Flanagan CH, deGraffenried LA and Hursting SD: The Role of the Insulin/IGF System in Cancer: Lessons Learned from Clinical Trials and the Energy BalanceCancer Link. Front Endocrinol (Lausanne) 6: 77, 2015.

15 Karlikova M, Topolcan O, Narsanska A, Kucera R, Treskova I and Treska V: Circulating Growth and Angiogenic Factors and Lymph Node Status in Early-stage Breast Cancer - A Pilot Study. Anticancer Res 36: 4209-4214, 2016.

16 Rohr I, Zeillinger R, Heinrich M, Concin N, Vergote I, Nassir M, Mahner S, E VANN, Trillsch F, Cacsire-Tong D, Chekerov R, Sehouli J and Braicu EI: Role of IGF-I in Primary Ovarian Cancer - A Study of the OVCAD European Consortium. Anticancer Res 36: 1015-1022, 2016.

17 Durzynska J: IGF axis and other factors in HPV-related and HPV-unrelated carcinogenesis (review). Oncol Rep 32: 22952306, 2014.

18 M'Hamdi H, Baizig NM, OE EL, M'Hamdi N, Attia Z, Gritli S, Gamoudi A, El May MV and May AE: Usefulness of IGF-1 serum levels as diagnostic marker of nasopharyngeal carcinoma. Immunobiology 221: 1304-1308, 2016.

19 Li J, Jin D, Fu S, Mei G, Zhou J, Lei L, Yu B and Wang G: Insulin-like growth factor binding protein-3 modulates osteoblast differentiation via interaction with vitamin D receptor. Biochem Biophys Res Commun 436: 632-637, 2013.

20 Renehan AG, Zwahlen M, Minder C, O'Dwyer ST, Shalet SM and Egger M: Insulin-like growth factor (IGF)-I, IGF binding protein-3, and cancer risk: systematic review and metaregression analysis. Lancet 363: 1346-1353, 2004.

21 Zhi X, Lamperska K, Golusinski P, Schork NJ, Luczewski L, Golusinski W and Masternak MM: Expression levels of insulinlike growth factors 1 and 2 in head and neck squamous cell carcinoma. Growth Horm IGF Res 24: 137-141, 2014.

22 Brady G, O'Regan E, Miller I, Ogungbowale A, Kapas S and Crean SJ: Serum levels of insulin-like growth factors (IGFs) and their binding proteins (IGFBPs), $-1,-2,-3$, in oral cancer. Int $\mathbf{J}$ Oral Maxillofac Surg 36: 259-262, 2007.

23 Wu X, Zhao H, Do KA, Johnson MM, Dong Q, Hong WK and Spitz MR: Serum levels of insulin growth factor (IGF-I) and IGF-binding protein predict risk of second primary tumors in patients with head and neck cancer. Clin Cancer Res 10: 39883995, 2004

24 Hung PS, Kao SY, Liu CJ, Tu HF, Wu CH and Lin SC: Insulinlike growth factor binding protein-5 enhances the migration and differentiation of gingival epithelial cells. J Periodontal Res 43: 673-680, 2008.

25 Psyrri A and DiMaio D: Human papillomavirus in cervical and head-and-neck cancer. Nat Clin Pract Oncol 5: 24-31, 2008.

26 Dang RP, Le VH, Miles BA, Teng MS, Genden EM, Bakst RL, Gupta V, Zhang DY, Demicco EG, Posner MR and Misiukiewicz KJ: Clinical outcomes in patients with recurrent or metastatic human papilloma virus-positive head and neck cancer. Anticancer Res 36: 1703-1709, 2016.

27 Allen CT, Lewis JS Jr., El-Mofty SK, Haughey BH and Nussenbaum B: Human papillomavirus and oropharynx cancer: biology, detection and clinical implications. Laryngoscope 120: 1756-1772, 2010.

28 Pickard A, Durzynska J, McCance DJ and Barton ER: The IGF axis in HPV associated cancers. Mutat Res Rev Mutat Res, 2017. http://doi.org/10.1016/j.mrrev.2017.01.002 [Epub ahead of print]
29 Kaklamani VG, Linos A, Kaklamani E, Markaki I and Mantzoros C: Age, sex, and smoking are predictors of circulating insulin-like growth factor 1 and insulin-like growth factor-binding protein 3. J Clin Oncol 17: 813-817, 1999.

30 Svensson J, Carlzon D, Petzold M, Karlsson MK, Ljunggren O, Tivesten A, Mellstrom D and Ohlsson C: Both low and high serum IGF-I levels associate with cancer mortality in older men. J Clin Endocrinol Metab 97: 4623-4630, 2012.

31 Tas F, Karabulut S, Serilmez M, Ciftci R and Duranyildiz D: Clinical significance of serum insulin-like growth factor-1 (IGF1) and insulinlike growth factor binding protein-3 (IGFBP-3) in patients with epithelial ovarian cancer. Tumour Biol 35: 31253132,2014

32 El Tayebi HM and Abdelaziz AI: Epigenetic regulation of insulin-like growth factor axis in hepatocellular carcinoma. World J Gastroenterol 22: 2668-2677, 2016.

33 Jung HJ and Suh Y: Regulation of IGF -1 signaling by microRNAs. Front Genet 5: 472, 2014.

$34 \mathrm{Yu} \mathrm{H}$ and Rohan T: Role of the insulin-like growth factor family in cancer development and progression. J Natl Cancer Inst 92: 1472-1489, 2000.

35 Durzynska $\mathbf{J}$ and Barton E: IGF expression in HPV-related and HPV-unrelated human cancer cells. Oncol Rep 32: 893-900, 2014.

36 Kasprzak A and Adamek A: The insulin-like growth factor (IGF) signaling axis and hepatitis $\mathrm{C}$ virus-associated carcinogenesis (review). Int J Oncol 41: 1919-1931, 2012.

37 Adamek A, Kasprzak A, Mikos H, Przybyszewska W, SeraszekJaros A, Czajka A, Sterzynska K and Mozer-Lisewska I: The insulin-like growth factor-1 and expression of its binding protein 3 in chronic hepatitis $\mathrm{C}$ and hepatocellular carcinoma. Oncol Rep 30: 1337-1345, 2013.

38 Chun YS, Huang M, Rink L and Von Mehren M: Expression levels of insulin-like growth factors and receptors in hepatocellular carcinoma: a retrospective study. World J Surg Oncol 12: 231, 2014.

39 Schaffer A, Koushik A, Trottier H, Duarte-Franco E, Mansour N, Arseneau J, Provencher D, Gilbert L, Gotlieb W, Ferenczy A, Coutlee F, Pollak MN and Franco EL: Insulin-like growth factorI and risk of high-grade cervical intraepithelial neoplasia. Cancer Epidemiol Biomarkers Prev 16: 716-722, 2007.

40 Mannhardt B, Weinzimer SA, Wagner M, Fiedler M, Cohen P, Jansen-Durr P and Zwerschke W: Human papillomavirus type 16 E7 oncoprotein binds and inactivates growth-inhibitory insulin-like growth factor binding protein 3. Mol Cell Biol 20: 6483-6495, 2000.

41 Polanska H, Heger Z, Gumulec J, Raudenska M, Svobodova M, Balvan J, Fojtu M, Binkova H, Horakova Z, Kostrica R, Adam V, Kizek R and Masarik M: Effect of HPV on tumor expression levels of the most commonly used markers in HNSCC. Tumour Biol 37: 7193-7201, 2016.

42 Kundu SK and Nestor M: Targeted therapy in head and neck cancer. Tumour Biol 33: 707-721, 2012.

43 Brahmkhatri VP, Prasanna C and Atreya HS: Insulin-like growth factor system in cancer: novel targeted therapies. Biomed Res Int 2015: 538019, 2015.

Received April 2, 2017

Revised April 13, 2017

Accepted April 20, 2017 